Produto \& Produção, vol. 16 n.1, p. 81-99, mar. 2015

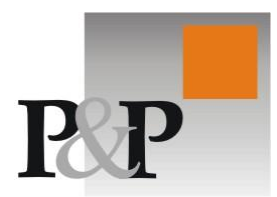

SUBMETIDO EM 04/09/2014. ACEITO EM 25/03/2015.

\title{
Proposição de um modelo de otimização para programação da produção em Sistema Flexível de Manufatura (FMS) com tempos de setup dependentes da sequência: a combinação de esforços em sequenciamento e tempos de preparação na indústria eletrônica
}

Proposition of a model for optimization to production scheduling in Flexible Manufacturing System (FMS) with sequence dependent setup time: a combination of efforts in sequencing and setup times in electronics industry

\section{Wagner Lourenzi Simões}

Universidade Luterana do Brasil - ULBRA

wlsjurai@hotmail.com

Rodrigo Dalla Vecchia

Universidade Luterana do Brasil - ULBRA

rodrigovecchia@gmail.com

\section{Macáliston Gonçalves DaSilva}

Universidade Luterana do Brasil - ULBRA

macaliston@ulbra.edu.br

\section{RESUMO}

Este trabalho tem como propósito pesquisar e auxiliar a programação da produção na indústria eletrônica, importante setor da economia. A pesquisa é desenvolvida em um Sistema Flexível de Manufatura (FMS - Flexible Manufacturing System) responsável pela montagem de placas de circuito impresso com tempos de setup dependentes da sequência de produção. O campo de estudo sugerido apresenta fatores comuns encontrados na manufatura de equipamentos eletrônicos caracterizados por trabalhar em ambientes HMLV (high-mix, low-volume). É proposto um modelo matemático visando minimizar o makespan, ou seja, a duração total de atendimento da programação, a partir da combinação de esforços em sequenciamento da produção e tempos de preparação. Os achados de pesquisa evidenciaram a possibilidade de utilização estratégica de duas lógicas de setup para execução da programação. A aplicação da alternância das estratégias, a partir dos desdobramentos da proposta de trabalho deste artigo, é o que gera a minimização do makespan.

Palavras-chave: Programação da Produção; Tempo s de Setup Dependentes da Sequência; Flow Shop Permutacional. 


\begin{abstract}
This paper aims to research and assist production scheduling in electronics industry, an important sector of the economy. The research is developed in a Flexible Manufacturing System (FMS) responsible for the assembly of printed circuit boards with sequence-dependent setup times. The field of study suggested presents common factors found in the manufacture of electronic equipment distinguished to work for HMLV (high-mix, low-volume) environments. A mathematical model to minimize the makespan, ie, the total length of service programming, from the combination of efforts in sequencing of production and setup times is proposed. Research findings showed the possibility of strategic use of two logic setup to implement scheduling. The alternation of strategies, according to the deployment of the work proposed in this paper, is what generates the minimization of makespan.
\end{abstract}

Key-words: Production Scheduling; Sequence-dependent Setup Times; Permutation Flow Shop.

\title{
1. Introdução
}

Hoto et al. (2010) destacam que a globalização da economia e o aumento da concorrência têm levado empresas a buscarem melhorias na qualidade e redução dos custos de produção com o emprego de variadas técnicas de otimização. A nova economia mundial, tal como citada por Hayes et al. (2008), combina três fatores: globalização; tecnologia avançada; e parcerias em rede. Os mesmos autores salientam a participação ativa da indústria eletrônica neste ambiente. É possível encontrar extenso material acadêmico sobre a indústria eletrônica internacional (DaSILVA, 2010), o que representa indício sobre a importância do segmento no cenário global, exemplificado por trabalhos de autores como Conti (2009), Doolen e Hacker (2005) e Dowdall et al. (2004).

Jabour e Jabour (2012) realçam a necessidade de avanços nas estratégias de gestão e condução das operações das empresas do segmento eletroeletrônico no Brasil. Fornecer alternativas eficientes para utilização dos recursos em suas atividades e sustentar a capacidade de flexibilidade do sistema produtivo parece ser pertinente para gestão da produção. Neste contexto encontra-se a atividade de programação da produção, uma das diversas funções executadas sob a responsabilidade do Planejamento e Controle da Produção (PCP).

A programação da produção envolve decisões de curto prazo e caracteriza-se como uma tarefa complexa no gerenciamento dos sistemas produtivos. Consiste em alocar no tempo as atividades segundo o sequenciamento definido em função das restrições percebidas, com o intuito de atingir um conjunto de objetivos estratégicos especificados pela empresa (SLACK et al., 2009; CORRÊA; CORRÊA, 2011). Entre outros resultados, a sua efetiva aplicação pode capacitar a empresa a obter produtos mais competitivos, otimizando o processo produtivo e reduzindo o lead time (ISHII et al., 2011). A procura pela eficiência dos recursos produtivos leva as empresas a buscarem além de alta velocidade e qualidade no processo operacional, a dominar a Tecnologia da Informação (TI), optando pelas melhores soluções em sistemas de gestão e controle de produção (REIS et al., 2012).

O tema Sistemas Flexíveis de Manufatura (FMS - Flexible Manufacturing System) tornou-se de extrema relevância nos últimos anos devido à concorrência acirrada na indústria de transformação, à redução do ciclo de vida dos produtos e ao tempo de resposta às necessidades dos clientes (RUIZ et al., 2009). Lee (2011) acrescenta que em resposta à concorrência no mercado global os Sistemas Flexíveis de Manufatura desempenham um papel importante para lidar com as rápidas mudanças. Estes sistemas, em geral, constituem-se por uma quantidade de máquinas-ferramentas e robôs tratando materiais, sistemas de armazenamento e de computadores (RUIZ, 2011).

Freire et al. (2013) diante destes fatores destacam que pode-se verificar que o FMS se diferencia de uma linha de produção tradicional devido a sua capacidade de produzir ao mesmo tempo mais de um tipo de produto, o que o torna flexível e produtivo, resultando no atendimento das flutuações do mercado. Para Deng e Yang (1999), o Sistema Flexível de Manufatura contribui para a melhoria da qualidade do produto, reduzindo os custos de produção e o tempo de espera.

O FMS é visto como um sistema complexo por causa de sua necessidade de acomodar variações das demandas, atender às exigências dos clientes e responder de forma imediata (BURNWAL; SANKHA, 2013). A introdução destes sistemas nos ambientes de manufatura proporciona vantagens, principalmente por lidar bem com as rápidas mudanças no mercado. Em 
pesquisa realizada por Freire et al. (2013), analisando estudos neste sentido, 42,2\% abordam o problema de programação com o objetivo de encontrar soluções que minimizem custos e maximizem o tempo de utilização de máquinas. Entretanto, os mesmos autores destacam que as atuais técnicas utilizadas em FMS vem enfrentando dois grandes problemas em sua aplicação: a modelagem e a complexidade.

Quanto a pesquisas envolvendo problemas de programação em flow shop (Flow Shop Scheduling Problem - FSSP), encontram-se trabalhos como o de Brucker e Shakhlevich (2011), que faz uma proposta de otimização inversa para o FSSP, de Li et al. (2012), que aborda flow shop com tempos dependentes da curva de aprendizado, de Navaei et al. (2013), que aborda o flow shop de dois estágios com o segundo estágio não idêntico, ou de Nip et al. (2015), que estuda uma cominação do FSSP com a lógica de sequenciamento por caminho mais curto. Porém, acompanhando Nejad et al. (2010), os métodos propostos na literatura para soluções à nível de programação da produção lidam principalmente com tarefas de processos em ambiente estático, no caso, onde as especificações do produto e do status do sistema de produção são estáveis. Assim, é perceptível a importância de trabalhos em ambientes dinâmicos, identificando as variabilidades do sistema, com a finalidade de desenvolver métodos ou ferramentas que sejam capazes de apresentar uma visão mais realista dos processos produtivos.

Este trabalho tem como propósito pesquisar e auxiliar a programação da produção na indústria eletrônica, mais especificamente, em um Sistema Flexível de Manufatura (FMS) responsável pela montagem de placas de circuito impresso (PCB - Printed Circuit Board) com tempo de preparação (setup) dependente da sequência de produção. Estes fatores são característicos de um ambiente HMLV (high-mix, low-volume), comumente encontrados na manufatura de equipamentos eletrônicos. $\mathrm{O}$ objetivo é propor um modelo matemático ao PCP para minimizar o makespan, ou seja, a duração total de atendimento da programação, a partir da combinação de esforços em sequenciamento da produção e tempos de setup.

Buscando uma fundamentação teórica para as ações de pesquisa apresenta-se, inicialmente, uma breve revisão da literatura, seguida da classificação e dos procedimentos metodológicos utilizados. A quarta seção trata da apresentação do problema e da formulação do modelo matemático associado ao processo de sequenciamento. As exposições dos aspectos relacionados à aplicação e à discussão dos resultados alcançados na situação investigada formam a quinta seção do artigo. Finalizase com as considerações finais e perspectivas de trabalhos futuros.

\section{Referencial teórico}

\subsection{Problemas de programação da produção}

Para Morais e Moccellin (2010), em problemas de programação da produção as restrições tecnológicas das atividades e os objetivos devem ser especificados, buscando com isso contribuições para diretrizes e métodos eficientes na utilização dos recursos em suas atividades. Allahverdi et al. (2008) e Morais e Moccellin (2010) descrevem a classificação de diversos tipos de problemas de programação a partir dos fluxos encontrados no ambiente produtivo, conforme segue:

- Job shop - cada tarefa tem sua própria ordem de processamento nos recursos produtivos;

- Flow shop - todas as tarefas têm o mesmo fluxo de processamento nos recursos produtivos;

- Open shop - não há especificação de fluxo para as tarefas serem processadas nos recursos produtivos;

- $\quad$ Flow shop permutacional - é o flow shop onde a ordem de processamento das tarefas deve ser a mesma em todos os recursos produtivos;

- $\quad$ Máquina única - existe apenas um recurso produtivo disponível;

- $\quad$ Máquinas paralelas - estão disponíveis mais de um recurso produtivo para as mesmas operações; 
- Job shop com múltiplas máquinas - é o job shop onde em cada estágio de processamento há um conjunto de recursos produtivos em paralelo;

- Flow shop com múltiplas máquinas - é o flow shop onde em cada estágio de processamento há um conjunto de recursos produtivos em paralelo.

Este trabalho aborda flow shop permutacional como problema de pesquisa.

\subsection{Influência dos tempos de setup sobre a eficiência do sistema produtivo}

Existe um crescente interesse em problemas de programação da produção envolvendo os tempos de setup. Este movimento está relacionado com o impacto positivo gerado nos mais diversos processos produtivos quando as decisões de programação incluem as variáveis de tempos de setup (ALLAHVERDI et al., 2008). Com o aumento da demanda por produtos eletrônicos, os fabricantes visam tornar seus sistemas produtivos mais eficientes, reduzindo os tempos de setup para melhor responder as necessidades dos consumidores, sendo na montagem de PCB, ambiente alvo desta pesquisa, as reduções dos tempos de setup associadas aos benefícios de reduções do tempo de atravessamento (lead time) e entrega, estoque em processo (work-in-process) e do custo de produção (SABOUNI; LOGENDRAN, 2013).

Apesar de avanços consideráveis na redução de tempos de setup, como o uso de Sistema Flexível de Manufatura (FMS) ou a metodologia Single-Minute Exchange of Die (SMED), ainda existem ambientes produtivos com tempos de preparação potenciais à eliminação (ALLAHVERDI $e t$ al., 1999). Em casos nos quais os tempos de setup apresentam razão significativa comparado com os tempos de processamento, há a possibilidade de tratamento diferenciado dos tempos de preparação no ato da programação da produção, dado a relação direta com a disponibilidade dos recursos, custos, atendimento à demanda e otimização do critério competitivo adotado (BARROS; MOCCELLIN, 2004).

Para a formulação de uma estratégia de setup, cabem as colocações de Flynn (1987), onde salienta que o tempo de duração da atividade de preparação está relacionado diretamente com o grau de similaridade entre as tarefas processadas sucessivamente. Segundo Wu e Ji (2010), a combinação de feeders, acessórios responsáveis pelo abastecimento automático de componentes eletrônicos, e o sequenciamento da produção são os dois problemas fundamentais de otimização na montagem de PCB. Em uma ordem sequencial, quanto maior a similaridade das tarefas a serem executadas, menor será o tempo requerido para o setup.

\subsection{Sequenciamento da produção com tempos de preparação dependentes}

Segundo Allahverdi et al. (1999), quando os tempos de setup dependem tanto da tarefa a ser executada como da tarefa processada imediatamente antes no mesmo recurso produtivo, este é chamado de tempo de preparação dependente da sequência (sequence-dependent). Caracteriza-se pelo fato dos tempos de setup para um mesmo recurso produtivo, após a execução de uma tarefa $j$, e para o processamento da tarefa $i$, ser diferente do tempo de preparação entre a mesma tarefa $j$ e outra tarefa qualquer, por exemplo, $k$ (BARROS; MOCCELLIN, 2004).

Barros e Moccellin (2004) exemplificam o impacto na programação com problemas de tempos de setup assimétricos e dependentes da sequência (ASDST - Asymmetric Sequence Dependent Setup Times). A Figura 1 mostra o tratamento de quatro tarefas considerando uma matriz de tempos de setup. 


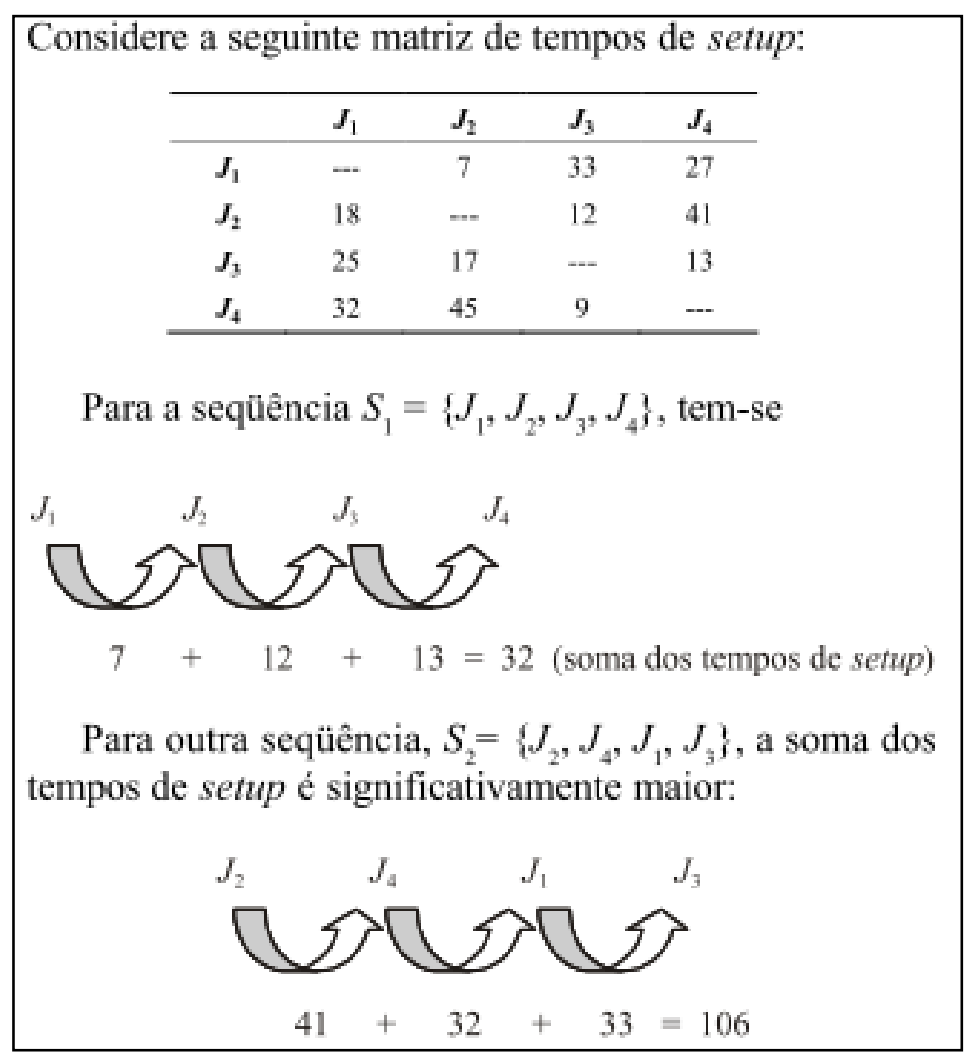

Figura 1 - Impacto dos tempos de setup assimétricos e dependentes da sequência.

Fonte: Barros e Moccellin (2004, p.103)

Este problema é abordado tradicionalmente com o modelo do Caixeiro Viajante (Traveling Salesman Problem - TSP). A tarefa do viajante é encontrar a mínima distância total necessária para visitar $n$ cidades, passando apenas uma vez por cada uma delas, e retornar à cidade de origem. No ponto de vista de sequenciamento, $s_{i j}$ representa o tempo de setup para a tarefa $j$ quando realizado imediatamente depois da tarefa $i$, correspondendo à distância entre as cidades $i$ e $j$, e encontrar a sequência de produção que atenda a todas as tarefas, mas minimize os tempos de preparação, equivale a encontrar um roteiro no TSP (PIZZOLATO et al., 1999).

No caso investigado, dado as características do ambiente dos negócios e do processo produtivo, existe a necessidade de tratamento das variáveis de tempo de setup na programação da produção. Na próxima seção apresentam-se as classificações e os procedimentos metodológicos adotados na pesquisa.

\subsection{Sequenciamento da produção em montagem de PCB}

Cyr et al. (1997) destacam o ambiente altamente dinâmico onde os fabricantes de produtos eletrônicos estão inseridos, caracterizado por demandas diversificadas de um mercado que se faz por evolução. Conceição et al. (2009) acrescentam que este dinamismo é alimentado pelo curto ciclo de vida dos produtos com altas taxas de inovações. Na busca por esta diferenciação uma variedade de trabalhos e estudos em sequenciamento de produção em fábricas de placa de circuito impresso pode ser encontrada na literatura internacional (e.g. WU; JI, 2010; HAIMING et al., 2009; KNUUTILA et al., 2004). Dentro deste universo uma grande gama estuda a utilização do arranjo de feeders para reduzir os tempos de preparação e de operação. Wu e Ji (2010) destacam que os ambientes produtivos de alta variedade de modelos colocam as empresas montadoras de placas de circuito impresso no desafio de frequentes setups envolvendo o rearranjo dos feeders fixados na máquina.

Alguns estudos, como Knuutila et al. (2004), Rosseti e Stanford (2003), buscam melhorar o agrupamento de montagens com maior similaridade em setup, tendo como objetivo reduzir as trocas necessárias. Outra vertente foca os estudos em um melhor arranjo dos feeders, de modo a se obter vantagens na otimização do curso de deslocamento da cabeça da máquina responsável por coletar os 
componentes nos feeders e depositá-los na devida posição na $\mathrm{PCB}$, reduzindo assim seu tempo de operação, como exemplos têm-se as pesquisas de Wu e Ji (2010), Ho e Ji (2003) e Sun et al. (2005).

Sabouni e Logendran (2013) corroboram com este levantamento, já que avaliaram diversas pesquisas envolvendo programação na montagem de PCB e identificaram um procedimento comum para abordagem dos problemas, primeiro tratar o grupo de componentes, depois o seu relacionamento com a família de PCBs (Quadro 1).

Quadro 1 - Estratégias para problemas de programação na montagem de PCB.

\begin{tabular}{|c|c|c|}
\hline Autor & Consideração Principal & Metodologia Coтит \\
\hline $\begin{array}{l}\text { McGinnis et al. } \\
\text { (1992) }\end{array}$ & $\begin{array}{l}\text { Foco na mudança de alimentadores de } \\
\text { componentes eletrônicos (feeders) }\end{array}$ & \multirow{5}{*}{$\begin{array}{l}\text { Todas as pesquisas assumiram } \\
\text { primeiramente o tratamento do } \\
\text { grupo de componentes, depois } \\
\text { relacionaram a família de PCBs } \\
\text { com o problema de } \\
\text { programação. }\end{array}$} \\
\hline $\begin{array}{l}\text { Tang e Denardo } \\
\text { (1988) }\end{array}$ & $\begin{array}{l}\text { Semelhante a investigação anterior, } \\
\text { porém com regras de restrições para as } \\
\text { trocas de feeders. }\end{array}$ & \\
\hline $\begin{array}{l}\text { Yilmaz e Günther } \\
\text { (2005) }\end{array}$ & $\begin{array}{l}\text { Ambiente de máquina única na } \\
\text { produção de lotes }\end{array}$ & \\
\hline $\begin{array}{l}\text { Ashayeri e Selen } \\
\text { (2007) }\end{array}$ & $\begin{array}{l}\text { Baseado na atribuição de componentes } \\
\text { para os feeders buscando minimizar as } \\
\text { trocas dos mesmos. }\end{array}$ & \\
\hline $\begin{array}{l}\text { Neammanne e } \\
\text { Reodecha (2009) }\end{array}$ & $\begin{array}{c}\text { Utilizou como ponderação nas } \\
\text { estratégias de setup a minimização do } \\
\text { atraso total. }\end{array}$ & \\
\hline
\end{tabular}

Fonte: Baseado em Sabouni e Logendran (2013)

\section{Metodologia}

É plausível se pensar que através da otimização do sequenciamento dos lotes de produção seja possível obter maior flexibilização em termos de variedade e de volume de produção. Isso seria resultante de um menor tempo de atravessamento dos lotes em uma linha de produção, sem demandar novos recursos. Baseado nesta lógica levanta-se a seguinte questão de pesquisa: como minimizar o tempo de atravessamento em um Sistema Flexível de Manufatura (FMS - Flexible Manufacturing System) responsável pela montagem de placas de circuito impresso (PCB - Printed Circuit Board) com tempo de preparação (setup) dependente da sequência de produção? Assim, o objetivo geral que norteia este trabalho é propor um modelo matemático para programação da produção em um Sistema Flexível de Manufatura responsável pela montagem de placas de circuito impresso a partir da combinação de esforços em sequenciamento da produção e tempos de preparação.

De acordo com os critérios de Manson (2006), o presente trabalho, em função do interesse prático que busca uma aplicação de resultado voltado à solução de um problema específico, classificase quanto à sua natureza como uma pesquisa aplicada. Para Cooper e Schindler (2001), a pesquisa aplicada em função de seu objetivo pragmático contribui para o desenvolvimento das organizações.

Como abordagem metodológica, inicialmente aplica-se uma pesquisa bibliográfica que se constitui no referencial teórico e teve como objetivo o aprofundamento dos pesquisadores nos temas relevantes para a investigação. A pesquisa de campo apoia-se nas bases conceituais do estudo de caso, para coleta de dados e aplicação dos achados em uma situação real, conforme orientações de Yin (2010) e Levy (2008). Acrescenta-se ainda nos passos investigativos a Pesquisa Operacional para embasar cientificamente o processo de tomada de decisão e formulação do modelo proposto.

Na pesquisa de campo, a coleta de informações dá-se através das técnicas de observação in loco, análise de documentos internos e entrevistas semiestruturadas com os gestores da empresa, a partir das indicações de Lakatos e Marconi (2011). De acordo com Yin (2010), a coleta de dados oriunda de diferentes fontes objetiva a triangulação de dados, permitindo maior amplitude e compreensão da situação estudada, além de conclusões mais robustas. 


\section{Descrição do caso}

O desenvolvimento do estudo foi realizado em uma empresa fabricante de produtos eletrônicos equipada com Sistema Flexível de Manufatura (FMS). O processo produtivo foco da investigação constitui-se de uma linha de montagem SMT (Surface Mount Technology) caracterizada pelo uso da tecnologia de inserção automática de componentes SMD (Surface Mount Design) na superfície de placas de circuito impresso (PCB - Printed Circuit Board).

As placas de circuito impresso são a matéria-prima básica da indústria eletrônica. A PCB é produzida em compósitos de fibra ou fenólicos não condutores com circuitos de cobre impressos em sua superfície. Estas placas constituem a base estrutural do circuito, onde são soldados os componentes eletrônicos.

Os componentes eletrônicos podem ser divididos em duas categorias: os componentes SMD (Surface Mounted Device) e os PTH (Pin Through Hole). Os componentes mais tradicionais são os PTH, também conhecidos popularmente como "convencionais" por terem sido durante muitas décadas a única opção. Os componentes SMD surgiram na metade dos anos 1960 a partir da necessidade de miniaturização dos produtos eletrônicos, em geral, estes componentes possuem dimensões menores que os PTH.

Uma das principais características dos componentes SMD no processo de manufatura é a soldagem na superfície da PCB, sem exigir furação para fixação do componente. Esta tecnologia de montagem é conhecida como SMT.

A montagem dos componentes SMD na indústria eletrônica ocorre, geralmente, através do processo de inserção automática, sendo os componentes posteriormente soldados pelo processo de refusão da pasta de solda, ou através do processo de soldagem por onda. Holcomb (1995) destacou em seu trabalho que a manufatura de produtos eletrônicos exige agilidade e completa confiabilidade, o que só pode ser obtida com uso de maquinário automatizado. A Figura 2 ilustra a configuração da linha de produção pesquisada.

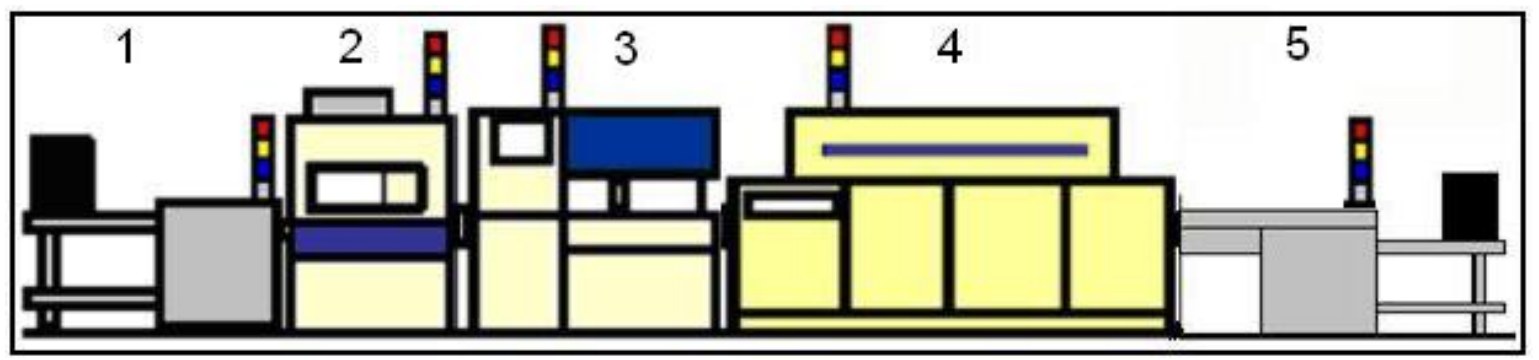

Figura 2 - Linha de produção SMT.

Fonte: Autores

O processo produtivo automatizado da empresa objeto de estudo é formado basicamente por cinco etapas:

1. Abastecedor (Loader) - responsável por alimentar a linha de produção com PCB, comunica-se com a próxima máquina da sequência alimentando-a automaticamente. Pode ser abastecido pelo operador através de racks ou pilhas de placas, no último caso, faz uso de ventosas pneumáticas para posicionar a PCB;

2. Screen Printer - responsável por depositar pasta de solda ou adesivo sobre as áreas de soldagem ou montagem dos componentes na PCB através do uso de stenceis. Possui comando automático para alimentação da próxima máquina, assim como solicita abastecimento para a máquina anterior;

3. Insersora (P\&P - Pick-and-Place) - responsável por inserir os componentes eletrônicos na PCB. Possui comando automático para alimentação da próxima máquina com execução da operação principal, assim como solicita abastecimento para a máquina anterior;

4. Forno (Reflow Oven) - responsável pela refusão da pasta de solda, e em alguns casos a cura de adesivo, e consequente fixação dos componentes eletrônicos na PCB. Possui múltiplas zonas de aquecimento controladas eletronicamente que em conjunto com o controle de velocidade do transporte efetuam o aquecimento das placas e soldagem dos componentes; 
5. Área de recolhimento da PCB montada.

Um FMS pode ser definido como um grupo de equipamentos ou células de manufatura ligadas por um sistema manipulador ou movimentador de material e outros equipamentos auxiliares, controlados por computador sendo capazes de processar lotes de diversos tamanhos, especialmente médio e baixo volume, bem como uma gama variada de modelos diferentes de produtos a custos consideravelmente reduzidos devido ao seu alto nível de automação, sendo comum a utilização de máquinas de comando numérico e robôs em sua constituição (LIU; YE, 2011; QIANLIANG, 2011; YUSOF et al., 2011). Esta linha de produção SMT em análise caracteriza-se como um FMS, pois o transporte entre as operações é realizado automaticamente e a manipulação do material ao longo das operações que a compõem é executada automaticamente, também. A programação e o controle do sistema podem ser feitos de forma centralizada via computador remoto e software de gerência. De maneira geral, é utilizado apenas um ou poucos operadores na linha, tendo como responsabilidade o controle de alguns parâmetros, abastecimento de pasta de solda na Screen Printer, abastecimento e descarga de PCB, ou seja, atividades auxiliares ao processo de produção automatizado

Os diferenciais da empresa no mercado são oferecer um alto grau de customização nos produtos, adequando-os às exigências e necessidades dos clientes, e um alto nível de flexibilidade na entrega. Ao mesmo tempo em que esses aspectos se mostram como fundamentais, geram custos maiores de produção decorrentes do alto mix de produtos com baixo volume de produção. Na operação, a flexibilidade exigida acarreta no acúmulo de setups ao longo do dia, atingindo um valor médio de oito por turno de trabalho. Atualmente, cada setup dura em média 25 minutos, resultando em cerca de 3 horas de atividades de setup para cada turno de 8 horas, o que representa uma perda de $37 \%$ no tempo efetivo de produção (up time).

O histórico da empresa mostra que a razão entre os tempos de setup e os tempos de processamento dos lotes é significativamente alta. O ponto mais crítico na produção é a troca de setup na $\mathrm{P} \& \mathrm{P}$, pois o modelo com o qual a empresa conta caracteriza-se por ser uma máquina de média performance e baixa flexibilidade, comparada com as necessidades da empresa. A maior parte do tempo de preparação é com atividades de setup interno, ou seja, com a máquina parada. O fato impacta diretamente no tempo de atravessamento dos lotes (lead time). Na tentativa de compensar o tempo gasto com setup, muitas vezes o PCP aumenta o tamanho do lote sem vincular o volume programado com a demanda direta prevista. Atualmente, a troca da máquina por outra com configuração de altíssima flexibilidade em setup, diminuindo drasticamente os tempos de preparação, é inviável financeiramente.

Sendo assim, o ponto mais crítico na produção é a troca de setup na Pick-and-Place (P\&P), tendo a substituição dos alimentadores (feeders) como elemento de tempo mais expressivo do processo. Os alimentadores são acessórios responsáveis pelo abastecimento automático de componentes eletrônicos na P\&P. Cada alimentador é municiado com um rolo de componentes SMD especificado pelo software de controle da máquina, que processa a estrutura de produto e coordenadas de montagem, e deve ser alocado em uma determinada posição na mesa de alimentação da P\&P, também apontada pelo software. Cada tipo de produto montado tem suas configurações particulares. Há alimentadores reservas, ou seja, que não estão em uso no momento da montagem. Os mesmos são alocados em um carrinho com uma peça idêntica a mesa de alimentação da máquina permitindo a verificação de disponibilidade de componentes e execução de preparação externa, sempre que possível.

Os alimentadores são universais, podem ser utilizados na produção de qualquer produto. Com a utilização do recurso de otimização automática de montagem, disponível no software de controle da $\mathrm{P} \& \mathrm{P}$, os alimentadores, em geral, precisam ser trocados de posição na mesa de alimentação a cada alteração de produto na linha. Nos casos onde o software exige um determinado componente que não está municiado naquele momento, ou a preparação se dá a partir de um alimentador livre, ou é necessário o desmonte de um alimentador já carregado para municiá-lo com o componente solicitado.

Cabe salientar que não há alimentadores reservas suficientes para preparação externa completa na grande maioria das vezes. $O$ investimento na compra de mais peças sobressalentes é financeiramente inviável para a empresa. 


\subsection{Formulação do modelo proposto e suas restrições}

A otimização de processos pode ser efetuada em diferentes níveis de atuação, tais como logística, projetos, operações, e por meio de diferentes procedimentos de aplicação (MOREIRA, 2010). Independente do nível e do tipo de aplicação, na busca por uma programação matemática, três são os aspectos fundamentais: a criação de uma função objetivo, a escolha das variáveis de decisão e a apresentação do conjunto de restrições (BRONSON, 1985).

É prática recorrente na literatura a aplicação de métodos de agrupamento e sequenciamento dos setups buscando-se tirar proveitos desta similaridade para promover a redução dos tempos de setup entre um produto e outro desta sequência. Como exemplos, se pode citar trabalhos como Gongaware e Ham (1984), Ham et al. (1985) e Wemmerlov e Vakharia (1991). O modelo proposto neste trabalho se embasa neste raciocínio de agrupamento por similaridade de setup. Sugere-se que as famílias sejam agrupadas pela similaridade dos componentes inseridos, atacando sempre em primeiro plano a quantidade de alimentadores que precisam ser trocados de um produto para outro, objetivando com isso a minimização do tempo de setup.

Rosseti e Stanford (2003) apontam que diversos trabalhos voltados ao sequenciamento da montagem de PCB utilizam os mais variados métodos, como branch and bound, formulações em programação inteira e uma grande variedade de heurísticas estáticas e dinâmicas. Buscando a determinação do melhor sequenciamento possível, construiu-se um modelo matemático que possui características de um problema de designação, diferenciando-se dos casos mais comuns por ser composto por uma função objetivo não-linear, segundo conceitos de Moreira (2010). Para composição do modelo estipulou-se como padrão a execução do cálculo para $n$ produtos com $n-1$ trocas de setup. Com base nisso, construiu-se uma matriz quadrada $M_{i j}$ para tomada de decisão.

$$
M_{i j}=\left[\begin{array}{ccccc}
x_{11} & x_{12} & x_{13} & \ldots & x_{1 n} \\
x_{21} & x_{22} & x_{23} & \ldots & x_{2 n} \\
x_{31} & x_{32} & x_{33} & \ldots & x_{3 n} \\
\vdots & \vdots & \vdots & & \vdots \\
x_{n 1} & x_{n 2} & x_{n 3} & \ldots & x_{n n}
\end{array}\right]
$$

Figura 3 - Matriz para tomada de decisão. Fonte: Autores

Nessa matriz, a i-ésima linha representa a i-ésima operação a ser efetuada e a j-ésima coluna representa o j-ésimo tipo de produto. A cada elemento dessa matriz de ordem $4 \times 4$ foi atribuído uma variável de decisão $x_{i j}$ com $i, j=1,2,3,4, \ldots, n$ que se caracteriza como dicotômica ou binária (DAGHLIAN, 1995), por assumir apenas dois estados, opção escolhida e opção não escolhida, que são representados no modelo matemático por 1 e 0 , respectivamente. Desse modo tem-se:

- $\quad$ Se $x_{i j}=0$ então a j-ésima peça não deverá ser a i-ésima a ser processada.

- $\quad$ Se $\quad x_{i j}=1$ então a j-ésima peça deverá ser a i-ésima a ser processada.

A Figura 4 mostra a relação entre os $n$ tipos de produtos e suas respectivas ordens de entrada na linha de produção. 


$$
\begin{array}{ccccccc} 
& A_{1} & A_{2} & A_{3} & A_{4} & \ldots & A_{n} \\
1^{\mathbf{o}} & x_{11} & x_{12} & x_{13} & x_{14} & \ldots & x_{1 n} \\
2^{\mathbf{o}} & x_{21} & x_{22} & x_{23} & x_{24} & \ldots & x_{2 n} \\
3^{\mathbf{o}} & x_{31} & x_{32} & x_{33} & x_{34} & \ldots & x_{3 n} \\
4^{\mathbf{o}} & x_{41} & x_{42} & x_{43} & x_{44} & \ldots & x_{4 n} \\
\vdots & \vdots & \vdots & \vdots & \vdots & & \vdots \\
n^{\mathbf{o}} & x_{n 1} & x_{n 2} & x_{n 3} & x_{n 4} & \ldots & x_{n n}
\end{array}
$$

Figura 4 - Relação entre produtos e a ordenação da produção.

Fonte: Autores

Associado a cada variável de decisão está outra informação que indica o tempo de setup gasto na mudança de uma peça a outra, definidos pela expressão $t_{i j} \operatorname{com} i, j=1,2,3,4, \ldots, n$ e $i \neq j$, onde $t_{i j}$ é o tempo de troca dos alimentadores da peça $i$ para a peça $j$. Para os casos onde $i=j$, utilizou-se $t_{i j}=0$. Optou-se por este recurso ao invés de simplesmente não considerar a variável pois, além de não haver perda de generalidade, pode facilitar o processo de programação do modelo, como ocorreu neste estudo. A Figura 5 mostra a relação entre todos os tipos de peças consideradas para o problema.

$$
\begin{array}{ccccccc} 
& A_{1} & A_{2} & A_{3} & A_{4} & \ldots & A_{n} \\
A_{1} & 0 & t_{12} & t_{13} & t_{14} & \ldots & t_{1 n} \\
A_{2} & t_{21} & 0 & t_{23} & t_{24} & \ldots & t_{2 n} \\
A_{3} & t_{31} & t_{32} & 0 & t_{34} & \ldots & t_{3 n} \\
A_{4} & t_{41} & t_{42} & t_{43} & 0 & \ldots & t_{4 n} \\
\vdots & \vdots & \vdots & \vdots & \vdots & & \vdots \\
A_{n} & t_{n 1} & t_{n 2} & t_{n 3} & t_{n 4} & \ldots & 0
\end{array}
$$

Figura 5 - Tempo de setup.

Fonte: Autores

Tendo em vista que parte do objetivo do trabalho consiste em reduzir o tempo total de setups realizados, criou-se uma Função Objetivo (F.O.) que determina esse valor. Para tanto, foram consideradas as trocas de setup, a saber:

Troca da primeira para a segunda peça.

$$
\mathrm{S}_{1}=\sum_{K=1}^{n}\left[x_{2 k} * \sum_{j=1}^{n}\left(x_{1 j} * t_{j k)}\right]\right.
$$

- $\quad$ Troca da segunda para a terceira peça.

$$
\mathrm{S}_{2}=\sum_{k=1}^{n}\left[x_{3 k} * \sum_{j=1}^{n}\left(x_{2 j} * t_{j k}\right)\right]
$$

- $\quad$ Troca da n-1-ésima para a n-ésima peça

$$
\mathrm{S}_{\mathrm{n}-1}=\sum_{k=1}^{n}\left[x_{4 k} * \sum_{j=1}^{n}\left(x_{3 j} * t_{j k}\right)\right]
$$


Nota-se que a multiplicação da soma dos produtos de tempo e decisão de cada linha da matriz $M_{i j}$ em cada uma das funções $S_{1}, S_{2}, \ldots, S_{n-1}$, pelo elemento de decisão da linha seguinte, permite que apenas a função possuidora deste multiplicador igual a 1 terá seu valor de tempo acrescido no somatório final da Função Objetivo. A Função Objetivo é dada pelo somatório dos tempos de troca de setup. Minimizando o valor dessa função obtém-se o melhor sequenciamento dos lotes, levando ao menor makespan. Generalizando o modelo, considerando-se que $n$ é a quantidade total de produtos que compõem a sequência de montagem, pode-se assumir a Função Objetivo como sendo:

$$
\text { F. O. Minimizar } \sum_{p=2}^{n}\left\{\sum_{k=1}^{n}\left[x_{p k} * \sum_{j=1}^{n}\left(x_{(p-1) j} * t_{j k}\right)\right]\right\}
$$

Com j, k, p $=1,2,3,4, \ldots, n$

Para garantir a unicidade na designação, que terá como consequência o sequenciamento da produção, utilizam-se as seguintes restrições:

$$
\begin{array}{ll}
\sum_{j=1}^{n} x_{i j}=1 & \text { para } i \in\{1,2,3,4, \ldots, n\} \text { com } x_{i j} \text { binário } \\
\sum_{i=1}^{n} x_{i j}=1 & \text { para } j \in\{1,2,3,4, \ldots, n\} \text { com } x_{i j} \text { binário }
\end{array}
$$

Estas restrições combinadas garantem que cada produto, necessariamente, será produzido uma única vez (1) e que não haverá repetições quanto à cardinalidade do sequenciamento (2). O modelo desenvolvido engloba apenas restrições de sequência, uma vez que trata-se de uma abordagem que visa a redução do makespan através da otimização da sequência, valendo-se das similaridades entre os produtos. Outras restrições inerentes ao campo industrial, como faltas de componentes na produção, paradas para manutenção de equipamentos, perdas no processo ou erros operacionais, não foram consideradas na construção deste modelo por entender-se que nesta fase do trabalho suas inclusões extrapolariam o escopo proposto. No caso, obter uma ferramenta de apoio à decisão que possibilite menor makespan possível através da redução do tempo de paradas por setup, valendo-se das similaridades entre as trocas. O incremento de tais restrições ao modelo tende a elevar o esforço computacional necessário, o que poderia inviabilizar a sua aplicação no contexto pesquisado, não resultando em uma ferramenta adequada as condições normais oferecidas pela empresa. Entende-se que as perdas no sistema produtivo, como no processo de preparação, por exemplo, devam ser priorizadas e tratadas na empresa objeto de estudo com os devidos esforços, buscando avanços sistêmicos. Neste sentido, parte-se da ideia que qualquer melhoria no processo produtivo pode ser convertida em novos valores assumidos pelas variáveis do modelo proposto.

\section{Aplicação e discussão dos resultados}

Uma vez estabelecido o modelo, buscou-se uma ferramenta computacional adequada para executar os cálculos necessários. Optou-se pelo uso do aplicativo Solver do Microsoft Office Excel 2007, software disponível e já utilizado pela empresa investigada. A Figura 6 mostra a interface gráfica definida. 


\begin{tabular}{|c|c|c|c|c|c|c|c|c|c|c|}
\hline$x$ & A & B & C & D & & $T$ & A & B & C & D \\
\hline 18 & 1 & & & & & A & $x$ & 9000 & 260 & 260 \\
\hline $2^{\circ}$ & & & & 1 & & B & 910 & $\mathrm{x}$ & 100 & 100 \\
\hline 39 & & 1 & & & & C & 3200 & 2470 & $\mathrm{x}$ & 340 \\
\hline 49 & & & 1 & & & D & 2467 & 786 & 349 & $\mathrm{x}$ \\
\hline F.O Min & & & & & \multirow{4}{*}{$\begin{array}{cc}\alpha & \text { troca } \\
1^{2} \text { para } 29\end{array}$} & 0,00 & & & & \\
\hline \multicolumn{3}{|l|}{ Sujeito a } & & & & 0,00 & & \multirow{2}{*}{ RESET } & & \\
\hline 1 & $=$ & 1 & & & & 0,00 & & & & \\
\hline 1 & $=$ & 1 & & & & 260,00 & & \multirow[b]{2}{*}{ TESTE 1} & & \\
\hline 1 & $=$ & 1 & & & \multirow{4}{*}{$\mid \begin{array}{c}\beta \text { troca } 22 \\
\text { para } 32\end{array}$} & 0,00 & & & & \\
\hline 1 & $=$ & 1 & & & & 786,00 & & \multirow{3}{*}{ DIAG. $P$} & & \\
\hline 1 & $=$ & 1 & & & & 0,00 & & & & \\
\hline 1 & $=$ & 1 & & & & 0,00 & & & & \\
\hline 1 & $=$ & 1 & & & \multirow{4}{*}{$\begin{array}{c}y \text { troca } \\
\text { do } 32 \\
\text { para } 42\end{array}$} & 0,00 & & \multirow{2}{*}{ DIAG.S } & & \\
\hline 1 & $=$ & 1 & & & & 0,00 & & & & \\
\hline & & & & & & 100,00 & & & & \\
\hline & & & & & & 0,00 & & & & \\
\hline
\end{tabular}

Figura 6 - Interface de trabalho.

Fonte: Autores

Para viabilizar os múltiplos cálculos do Solver durante os testes das várias condições iniciais necessárias, foram implementadas Macros na planilha do modelo, conforme recomendam Powell e Baker (2006), buscando minimizar possíveis erros frente às distinções entre mínimo absoluto e mínimo local, utilizando-se os procedimentos de multiplicidade inicial, descritos por Lachtermacher (2009). Dessa forma, o preenchimento das condições iniciais acontece automaticamente, possibilitando que sucessivos cálculos sejam executados de maneira ágil.

Objetivando otimizar os tempos de setup buscou-se uma reestruturação no modo como os alimentadores são dispostos na mesa de alimentação, ou seja, na lógica de distribuição física dos feeders na $\mathrm{P} \& \mathrm{P}$. Originalmente os alimentadores são alocados a partir de um mapa gerado pelo software de controle da P\&P em função da lógica de otimização do curso automático da máquina, tendo como base a PCB a ser montada e visando reduzir o deslocamento de pega dos componentes de maior utilização no processo de produção, minimizando perdas de produtividade. A iniciativa de reestruturação procurou seguir as ideias de similaridade propostas por Flynn (1987). Seguindo essas orientações, condicionou-se que as posições de cada alimentador na mesa de alimentação da P\&P fossem fixadas em ordem decrescente de ocorrência de solicitação do componente pelas estruturas de produtos ativos. Fisicamente, partiu-se do local mais próximo onde a PCB é presa no interior da máquina. Buscou-se com isso possibilitar que componentes comuns a dois ou mais produtos não tenham suas posições alteradas quando montados em sequência. Os softwares de controle da máquina, referentes aos produtos programados, foram reconfigurados de forma a ignorarem a otimização de curso automático oferecido pelo equipamento, passando a utilizar a lógica mesa de alimentação fixa. É importante frisar que esta ação reduz a performance do equipamento durante o processo de inserção, aumentando o tempo de processamento das PCBs. Porém dada a característica de produção discreta em lotes, com baixos volumes, entende-se que existem casos nos quais os ganhos com a redução de setup superam as perdas de performance.

Com a construção do modelo e parametrização no aplicativo Solver, iniciou-se a fase de testes a fim de se obter validação preliminar da funcionalidade da proposta, bem como, relevância para aplicação na programação da produção. Foram selecionados quatro produtos com características de montagem distintas. Na Tabela1 têm-se os dados obtidos após produção com uso do modelo de sequenciamento e a fixação dos alimentadores. Nesse caso, observa-se que o tempo de processamento foi afetado pelo acréscimo no deslocamento até os alimentadores. Em contrapartida, a hipótese esperada de redução do tempo de setup foi confirmada, reduzindo em $29 \%$ o tempo médio destinado para troca de produtos na linha de produção. Avaliando os dados tabelados é possível notar que a utilização do modelo matemático de sequenciamento, juntamente com a estratégia de fixação dos alimentadores, proporcionou a redução de $25,41 \%$ no tempo total de finalização dos lotes, o 
makespan, passando de 81,37 minutos para 60,69 minutos. Observa-se também que a relação existente entre a redução dos tempos de setup e o aumento do tempo de processamento se mostrou vantajosa.

Tabela 1- Resultados do teste do modelo.

\begin{tabular}{|c|c|c|c|c|c|c|}
\hline Ordem & Produto & $\begin{array}{c}\text { Placas por } \\
\text { Painel }\end{array}$ & $\begin{array}{c}\text { Tempo de } \\
\text { Processamento } \\
\text { CA* } \\
\text { (min/painel) }\end{array}$ & $\begin{array}{c}\text { Tempo } \\
\text { Médio de } \\
\text { Setup CA* } \\
\text { (min) }\end{array}$ & $\begin{array}{c}\text { Tempo de } \\
\text { Processamento } \\
\text { MF** } \\
\text { (min/painel) }\end{array}$ & $\begin{array}{c}\text { Tempo } \\
\text { Médio de } \\
\text { Setup MF* } \\
\text { (min) }\end{array}$ \\
\hline $1^{\circ}$ & SC-W555 & 4 & 3,33 & \multirow{4}{*}{25,00} & 3,95 & \multirow{4}{*}{17,75} \\
\hline $2^{\circ}$ & SC-D301 & 10 & 2,62 & & 2,97 & \\
\hline $3^{\circ}$ & SC-TS510 & 1 & 0,25 & & 0,32 & \\
\hline $4^{\circ}$ & SC-T047 & 1 & 0,17 & & 0,20 & \\
\hline \multicolumn{2}{|c|}{$\overline{M a k e s p a n}(\min )$} & & 81,37 & & 60,69 & \\
\hline
\end{tabular}

$C A^{*}$ - lógica de otimização com curso automático

$M F^{*}$ - lógica de otimização com mesa de alimentação fixa

Fonte: Autores

Cabe salientar que, embora na especificidade da situação analisada o aumento do tempo de processamento em função da diminuição do tempo de setup referente ao processo de fixação de alimentadores tenha sido vantajosa, essa diferença positiva nem sempre ocorre, principalmente se a demanda de um determinado produto for alta. A redução do makespan é dependente da relação existente entre a redução dos tempos de setup e o aumento do tempo de processamento. Para impedir casos semelhantes onde incorre prejuízo ao tempo de fluxo total foi desenvolvida uma expressão matemática (3) que identifica o Ponto de Quebra da Lógica de Setup (PQLS). O PQLS está diretamente associado com a ideia de que para não haver nem perdas, nem ganhos de tempo, a diferença entre os tempos de processamento dos lotes nas duas lógicas de setup deve ser igual à diferença entre os tempos de setup das duas lógicas de trabalho. Desse modo, o PQLS pode ser definido pela equação:

$$
(P * T 2)-(P * T 1)=S 1-S 2
$$

Onde:

$P=$ Quantidade de painéis de PCB no lote;

$T 1$ = Tempo de processamento com otimização de curso automático;

$T 2=$ Tempo de processamento com a lógica mesa de alimentação fixa;

$S 1$ = Tempo de setup com otimização de curso automático;

$S 2$ = Tempo de setup com a lógica mesa de alimentação fixa.

A partir dessa expressão é possível encontrar uma função que define o tamanho de lote com perda nula (4). Para tanto, isola-se a variável que representa o tamanho de lote com perda nula. Ao multiplicá-lo pelo número de placas que cada painel comporta é possível se obter o tamanho do lote no Ponto de Quebra da Lógica de Setup.

$$
L=\left(\frac{S 1-S 2}{T 2-T 1}\right) * P c i
$$

Onde:

$L=$ Total de PCBs no PQLS ou Lote de Corte;

$P c i=$ Quantidade de placas montadas por painel.

O Lote de Corte $(L)$ servirá como balizador na tomada de decisões. Para programação de lotes maiores que o Lote de Corte é pertinente manter a utilização da estratégia de otimização de curso automático vigente na empresa. Já que, ao otimizar a sequência através da mesa fixa e do modelo proposto ganha-se o tempo da troca de posição dos alimentadores, porém, perde-se tempo ao fazer a 
cabeça de montagem da máquina percorrer um curso maior até a posição fixa do alimentador. Em lotes maiores que o Lote de Corte este tempo perdido em aumento do curso supera o tempo ganho com a redução de troca de posições dos alimentadores. Em lotes menores que o Lote de Corte é mais vantajosa a adoção da lógica mesa de alimentação fixa para os componentes, neste caso, ganha-se mais tempo com as mudanças de posição dos alimentadores que deixam de ocorrer, do que o aumento do tempo de montagem consequente do aumento do curso da cabeça de montagem.

\subsection{Algoritmo de escolha da lógica de sequenciamento}

Para se obter um melhor resultado possível no sequenciamento propõe-se um algoritmo que separa os produtos sequenciados em duas listas. De um lado $S a$, para os que possuem a quantidade acima do Lote de Corte, de outro Sm, para os lotes com a quantidade abaixo do Lote de Corte, direcionando os lotes com quantidade exatamente igual ao Lote de Corte, situação na qual não há vantagem de uma opção sobre a outra, para a lista que possua a mesma configuração do último lote montado. Cada uma destas listas deve ser otimizada através do modelo proposto anteriormente de forma isolada, e assim, define-se o melhor resultado em cada lista. Conhecendo a última montagem feita e sua propriedade quanto a lógica de setup, mesa fixa ou não, sequenciam-se as duas listas a partir desta informação, onde nas novas listas a primeira a ser executada seja aquela que obedeça a mesma lógica ou estrutura de setup que a última montagem.

Para facilitar a compreensão, propõem-se o algoritmo a seguir, onde:

$n=$ Produtos a serem sequenciados;

$C=$ Quantidade de produtos já sequenciados;

$Q=$ Tamanho de lote do produto selecionado para análise;

$L=$ Lote de Corte calculado para o produto selecionado para análise.

1.Inicio:

2. Faça $C=0$

2. Enquanto $C<\mathrm{n}$ faça:

3. Selecionar produto da lista à sequenciar ainda não analisado

4. $\quad$ Se $Q>L$ então:

5.

6.

7.

8.

9.

10.

11.

12.

13.

14.

15.

16.

17.

18.

Incluir produto na lista $\mathrm{Sa}$

\section{Fim se}

Se $(Q=L)$ então:

Se setup anterior foi montado com mesa fixa então:

Senão

Inclui produto na lista $\mathrm{Sm}$

Fim se

Incluir produto na lista $S a$

Fim se

19. Otimizar a sequência da lista $S a$

20. Otimizar a sequência da lista $\mathrm{Sm}$

21. Se setup anterior foi montado com mesa fixa então:

22. Iniciar produção pela sequência da lista $S m$

23. Senão

24. Iniciar produção pela sequência da lista $S a$

25. Fim se

26. Produzir a sequência da lista restante

27. Fim 
O sequenciamento das listas $S a$ e $S m$ permite resultados melhores por levar em consideração a estrutura de setup utilizada anteriormente. Pode ser visto como uma avaliação cíclica que sempre busca a maior similaridade possível entre o setup anterior e o próximo setup a ser utilizado.

\subsection{Implicações gerenciais}

De maneira a obter maior eficiência de seus sistemas produtivos as indústrias utilizam uma infinidade de regras de sequenciamento, sendo muitas delas já consagradas pela literatura, tais como, data prometida ou data devida, first in first out (FIFO), last in first out (LIFO), maior tempo de processo (MATP), menor tempo de processo (METP), regra de Johnson... (CORRÊA; CORRÊA, 2011; SLACK et al., 2009). Porém, todas estas regras de sequenciamento levam em consideração apenas fatores externos ao ambiente fabril, como data de entrega e data de entrada na produção, ou tratam os tempos de processo isoladamente sem aprofundar o quanto as relações de precedência entre os produtos podem afetar, por exemplo, os tempos de preparação.

O modelo proposto neste trabalho para o sequenciamento da produção considera as características comuns entre os produtos (componentes utilizados) e explora as relações de precedência, que se bem utilizadas possibilitam redução no tempo e nos custos de setup na produção, além de proporcionar sensível redução no makespan. Outro ponto, que a técnica desenvolvida para sequenciamento auxilia o sistema produtivo em se torne mais flexível quanto a volumes produzidos e variações no mix de produção. O sequenciamento e suas duas formas alternativas de organização da mesa de alimentação tornam o processo produtivo capaz de adaptar-se tanto a mix de produtos altamente variado e com baixos volumes quanto a mix de produtos pouco variado e com grandes volumes, extraindo sempre a melhor performance possível de ser obtida explorando as características dos lotes a serem montados.

Esta capacidade de lidar com mix e volumes de produção tão distintos utilizando o mesmo sistema produtivo e o modelo proposto de otimização para programação da produção pode gerar para empresa vantagens diferenciadas perante a concorrência, isso através da habilitação para competir em flexibilidade de volume e de modelos, inovação, prazo de entrega e custo, buscando sempre aderência as exigências dos clientes.

\section{Conclusão}

Ao fim dos experimentos verificou-se que o modelo sugerido por este estudo é factível, propondo um ferramental matemático ao PCP para minimizar o makespan, a partir da combinação de esforços em sequenciamento da produção e tempos de preparação. Os achados de pesquisa, no caso investigado, evidenciaram a possibilidade de utilização estratégica de duas lógicas de setup para execução da programação. Ou seja, caminhos alternativos para otimização do sequenciamento em função da variabilidade dos perfis de demanda e mix de produtos.

Levando-se em consideração o Lote de Corte e o prazo para entrega pode-se agrupar os lotes de produção por estratégia, permitindo dessa forma que a fábrica trabalhe com a lógica de otimização de curso automático em turnos com mix de produtos de baixa variedade e com altos volumes e com a lógica mesa de alimentação fixa em turnos com mix de produtos altamente variados e de baixos volumes. A aplicação da alternância das estratégias, a partir dos desdobramentos da proposta de trabalho deste artigo, é o que gera a minimização do makespan.

Os autores entendem que a pesquisa contribui para melhoria na tomada de decisão do PCP na produção de equipamentos eletrônicos caracterizados por trabalhar em Sistema Flexível de Manufatura (FMS - Flexible Manufacturing System) com tempo de preparação (setup) dependente da sequência de produção. Mas, a proposta deve ser vista como abordagem inicial de um processo evolutivo.

Este estudo e os resultados colhidos por sua aplicação no ambiente produtivo da empresa estudada podem subsidiar o desenvolvimento de soluções mais complexas, que poderão ser desenvolvidas futuramente, como o dimensionamento dos lotes baseados em parâmetros de estoque e histórico de demanda dos produtos, a automatização da geração de cenários de programação para agilizar o processo de tomada de decisão em função das estratégias de setup disponíveis, e o desenvolvimento de uma interface gráfica mais amigável para a operação, por exemplo. A inclusão de 
dados relativos a eficiência do FMS poderão propiciar também informações mais robustas e próximas a realidade do chão de fábrica ao tomador de decisão, fornecendo informações a respeito do tempo necessário para execução da sequência. Alguns outros parâmetros, especialmente relativos a demanda e disponibilidade, poderão resultar em melhor dimensionamento dos lotes, porém tendem a possuir comportamentos estocásticos, o que exigirá um maior esforço computacional para a solução do modelo. Dessa forma, desponta também como um importante campo para pesquisas futuras, o estudo de outras técnicas matemáticas para o tratamento do problema, como a aplicação de metaheurísticas para melhorar o desempenho computacional no processamento dos dados, o que por sua vez representará significativo incremento em termos de complexidade e de agregação de novas variáveis de decisão ao modelo. A evolução computacional pode abrir ainda estudos de combinação deste modelo com outras regras de sequenciamento relativas a aspectos que impactam diretamente na satisfação do cliente, como data prometida ou data devida. Este tipo de melhoria buscaria maior segurança e confiabilidade de entrega.

\section{Referências}

ALLAHVERDI, A.; GUPTA, J.N.D.; ALDOWAISAN, T. A review of scheduling research involving setup considerations. Omega - The International Journal of Management Science, v.27, n.2, p.219-239, April 1999.

ALLAHVERDI, A.; NG, C.T.; CHENG, T.C.E.; KOVALYOV, M.Y. A survey of scheduling problems with setup times or costs. European Journal of Operational Research, v.187, n.3, p.9851032, June 2008.

BARROS, A.D.; MOCCELLIN, J.V. Análise da flutuação do gargalo em flow shop permutacional com tempos de setup assimétricos e dependentes da seqüência. Gestão \& Produção, v.11, n.1, p.101108, 2004.

BRONSON, R. Pesquisa Operacional. São Paulo: McGraw-Hill do Brasil, 1985.

BURNWAL, S.; SANKHA, D. Scheduling optimization of flexible manufacturing system using cuckoo search-based approach. International Journal Advanced Manufacturing Technology, v.64, p.951-959, 2013.

BRUCKER, P. SHAKHLEVICH, N.V. Inverse scheduling: two-machine flow-shop problem. Journal of Scheduling, v.14, p.329-256, 2011.

CONCEIÇÃO, S.V.; RODRIGUES, I.A.; AZEVEDO, A.A.; ALMEIDA, J.F.; FERREIRA, F.; MORAIS, A. Desenvolvimento e implementação de uma metodologia para troca rápida de ferramentas em ambientes de manufatura contratada. Gestão \& Produção, v.16, n.3, p.357-369, 2009.

CONTI, J.P. Made in China - and designed there too. Engineering \& Technology, v.4, n.16, p.66-69, 2009.

COOPER, D.R.; SCHINDLER, P.S. Métodos de pesquisa em administração. Porto Alegre: Bookman, 2001.

CORRÊA, H.L.; CORRÊA, C.A. Administração da produção e operações - manufatura e serviços: uma abordagem estratégica. 2. ed. São Paulo: Atlas, 2011.

CYR, B.; LAMBERT, S.; ABDUL-NOUR, G.; ROCHETTE,R. Manufacturing flexibility: SMT factors study. Computers and Industrial Enegineering, v.33, n.1-2, p.361-364, 1997.

DAGHLIAN, J. Lógica e álgebra de Boole. São Paulo: Atlas, 1995.

DaSILVA, M.G. Avaliação do alinhamento entre critérios competitivos e práticas de autonomação na indústria eletrônica: um estudo de caso. São Leopoldo: UNISINOS, 2010. 124 p. Dissertação (Mestrado em Engenharia de Produção e Sistemas) - Universidade do Vale do Rio dos Sinos, São Leopoldo, 2010. 
DENG, Y.; YANG, C. Architecture-driven modeling of real-time concurrent systems with applications in FMS. Journal of Systems and Software, v.45, p.61-78, 1999.

DOOLEN, T.L.; HACKER, M.E. A review of lean assessment in organizations: na exploratory study of lean practices by electronics manufacturers. Journal of Manufacturing Systems, v.24, n.1, p.55$67,2005$.

DOWDALL, P.; BRADDON, D.; HARTLEY, K. The UK defence electronics industry: adjusting to change. Defence and Peace Economics, v.15, n.6, p.565-586, 2004.

FLYNN, B.B. The effects of setup time on output capacity in cellular manufacturing. International Journal of Production Research, v.25, n.12, p.1761-1772, 1987.

FREIRE, A.; MARINHO, E.S.; OLIVEIRA, S.C.R.; FAGUNDES, L.F.; ARAUJO, L.E.D. Sistemas flexíveis de manufatura: revisão bibliográfica sistemática. In: SIMPÓSIO NACIONAL DE ENGENHARIA DE PRODUÇÃO - XX SIMPEP, Anais... Bauru: UNESP, 2013.

GONGAWARE, T.A.; HAM, I. Cluster analysis applications for group technology manufacturing systems. In N. Lee, Group technology at work. Dearborn: Society of Manufacturing Engineers, 1984.

HAIMING, L.; PENG, Y.; JIAXIANG, L.; MEI, Z. Optimization algorithm for low-volume and highmix PCB assembly. Fifth International Conference on Natural Computation, p.379-383, 2009.

HAM, I.; HITOMI, K.; YOSHIDA, T. Group technology applications to production management. Boston, MA: Kluwer-Nijhoff, 1985.

HAYES, R.; PISANO, G.; UPTON, D.; WHEELWRIGHT, S. Em busca da vantagem competitiva: produção, estratégia e tecnologia. Porto Alegre: Bookman, 2008.

HO, W.; JI, P. A hybrid genetic algorithm for component sequencing and feeder arrangement. Journal of Intelligent Manufacturing, v.15, p.307-315, 2004.

HOLCOMB, G. W. Justifying flexible automation for PCB assembly. Assembly Automation, v.15, n.2, p.14-16, 1995.

HOTO, R.; BORSSOI, A.; MACULAN, N.; FENATO, A. Reducing the setup of a tubettes machine. IEEE Latin American Transactions, v.8, n.1, p.101-106, 2010.

ISHII, F.T.; GALDAME, E.V.C.; LEAL, G.C.L,; DORTA, R.P.; DIAS, A.L.L.S. Ensino de Planejamento e Controle da Produção como ferramenta para inclusão social. Gestão da Produção, Operações e Sistemas, v.6, n.4, p.157-167, 2011.

JABBOUR, A.B.L.S.; JABBOUR, C.J.C. Lançando luzes sobre a gestão de operações do setor eletroeletrônico brasileiro. Revista de Administração Pública, v.46, n.3, p.817-840, Maio/Jun. 2012.

KNUUTILA, T.; JOHNSSON, M.; NEVALAINEN, O. Grouping PCB assembly jobs with feeders of several types. The International Journal of Flexible Manufacturing Systems, v.16, p.151-167, 2004.

LACHTERMACHER, G. Pesquisa operacional na tomada de decisões. 4. ed. São Paulo: Pearson Prentice Hall, 2009.

LAKATOS, E.M.; MARCONI, M.A. Metodologia do trabalho científico. 7. ed. São Paulo: Atlas, 2011.

LEE, J.S. A modular command filtering approach to coordination of flexible manufacturing systems. The International Journal of Advance Manufacturing Technology, v. 56, n. 9-12, p.115-1123, 2011.

LEVY, J.S. Case studies: types, designs, and logics of inference. Conflict Management and Peace Science, v.25, n.1, p.1-18, 2008.

LI , G.; WANG, X.;WANG, J., SUN, L. Worst case analysis of flow shop scheduling problems with a time-dependent learning effect. International Journal of Production Economics, v.142, p.98-104, 2013. 
LIU, L.; YE, J. An approach to Scheduling of Flexible manufacturing System. IEEE International Conference on Electronic \& Mechanical Engineering and Information Technology, p.1777-1779, Harbin, 2011.

MANSON, N.J. Is operations research really research? Orion, v.22, n.5, p.155-180, 2006.

MORAIS, M.F.; MOCCELLIN, J.V. Métodos heurísticos construtivos para redução do estoque em processo em ambientes de produção flow shop híbridos com tempos de setup dependentes da sequência. Gestão \& Produção, v.17, n.2, p.367-375, 2010.

MOREIRA, D.A. Pesquisa operacional: curso introdutório. 2. ed. São Paulo: Cengage Learning, 2010.

NAVAEI, J.; GHOMI, S.M.T.; JOLAI, F.; SHIRAQAI, M.E.; HIDAJI, H. Two-stage flow-shop scheduling problem with non-identical second stage assembly machines. International Journal of Advanced Manufacturing Technology, v.69, p.2215-2226, 2013.

NEJAD, H.T.N.; SUGIMURA, N.; IWAMURA, K.; TANIMIZU, Y. Multi agent architecture for dynamic incremental process planning in the flexible manufacturing system. Journal of Intelligent Manufacturing, v.21, p.487-499, 2010.

NIP, K.; WHANG, Z.; TALLA, N.F.; LEUS, R. A combination of flow shop scheduling and the shortest path problem. Journal of Combinatorial Optimization, v.29, p.36-52, 2015.

PIZZOLATO, N.D.; VÁSQUEZ, S.G.G.; D’ÁVILA, S.L.G. O problema do seqüenciamento da produção em uma indústria química: avaliação de uma aplicação real. Gestão \& Produção, v.6, n.1, p.16-29, Abr. 1999.

POWEL, S.G.; BAKER, K.R. A arte da modelagem com planilhas: ciência da gestão, engenharia de planilhas e arte da modelagem. Rio de Janeiro: LTC, 2006.

QIANLIANG, C. The system development of FMS monitoring and fault diagnosis. IEEE International Conference on Electronic \& Mechanical Engineering and Information Technology, p.1777-1779, Harbin, 2011.

REIS, A.G.; TUBINO, D.F.; CAMPOS, P.C. Advanced Planning and Scheduling: com ênfase à produção de capacidade finita. Visão, v.1, n.1, p. 21-34, 2012.

ROSSETI, M.D.; STANFORD, K.J.A. Group sequencing a PCB assembly system via an expected sequence dependent setup heuristic. Computers \&Industrial Engineering, v.45, p.231-254, 2003.

RUIZ, M.C.; CAZORLA, D.; CUARTERO, F.; MACIA, H. Improving performance in flexible manufacturing systems. The Journal of Logic and Algebric Programming, v.78, n.4, p.260-273, 2009.

RUIZ, R. Book review of "Experimental Methods for the Analysis of optimization Algorithms". European Journal of Operational Researc,. v.214, n.2, p.453-456, 2011.

SABOUNI, M.T.Y.; LOGENDRAN, R. Carryover sequence-dependent group scheduling with the integration of internal and external setup times. European Journal of Operational Research, v.224, n.1, p.8-22, January 2013.

SLACK, N.; CHAMBERS, S.; JOHNSTON, R. Administração da produção. 3. ed. São Paulo: Atlas, 2009.

SUN, D.; LEE, T.; KIM, K. Component allocation and feeder arrangement for a dual-gantry multihead surface mounting placement tool. International Journal of Production Economics, v.95, n.2, p.245-264, 2005.

WEMMERLOV, U.; VAKHARIA, A. Job and family scheduling of a flow-line manufacturing cell: A simulation study. IIE Transactions, v.23, n.4, p.383-393, 1991. 
WU, Y.Z.; JI, P. Optimizing feeder arrangement of a PCB assembly machine for multiple boards. IEEE International Conference on Industrial Engineering and Engineering management. Macao: Institute of Electrical and Electronics Engineers, December, 2010.

YIN, R.K. Estudo de caso: Planejamento e Métodos. 4. ed. Porto Alegre: Bookman, 2010.

YUSOF, U.; BUDIARTO, R.; DERIS, S. Harmony Search algorithm for flexible manufacturing system (FMS) machine loading problem. IEEE 3rd Conference on Data Mining and Optimization (DMO). p.26-31, Selangor, 2011. 